\title{
SNPs in the KCNJ11-ABCC8 gene locus are associated with type 2 diabetes and blood pressure levels in the Japanese population
}

\author{
Yukiko Sakamoto $\cdot$ Hiroshi Inoue $\cdot$ Parvaneh Keshavarz $\cdot$ Katsuyuki Miyawaki · \\ Yuka Yamaguchi · Maki Moritani $\cdot$ Kiyoshi Kunika · Naoto Nakamura $\cdot$ Toshikazu Yoshikawa · \\ Natsuo Yasui · Hiroshi Shiota · Toshihito Tanahashi · Mitsuo Itakura
}

Received: 6 July 2007 / Accepted: 2 August 2007/Published online: 6 September 2007

(C) The Japan Society of Human Genetics and Springer 2007

\begin{abstract}
Many genetic association studies support a contribution of genetic variants in the KCNJ11-ABCC8 gene locus to type 2 diabetes (T2D) susceptibility in Caucasians. In non-Caucasian populations, however, there have been only a few association studies, and discordant results were obtained. Herein, we selected a total of 31 SNPs covering a 211.3-kb region of the KCNJ11-ABCC8 locus, characterized the patterns of linkage disequilibrium (LD) and haplotype structure, and performed a case-control association study in a Japanese population consisting of 909 T2D patients and 893 control subjects. We found significant associations between eight SNPs, including the KCNJ11 E23K and ABCC8 S1369A variants, and T2D.
\end{abstract}

Electronic supplementary material The online version of this article (doi:10.1007/s10038-007-0190-x) contains supplementary material, which is available to authorized users.

Y. Sakamoto $\cdot$ H. Inoue $(\bowtie) \cdot$ P. Keshavarz · K. Miyawaki ·

Y. Yamaguchi · M. Moritani - K. Kunika - T. Tanahashi ·

M. Itakura

Division of Genetic Information, Institute for Genome Research,

The University of Tokushima, \#3-18-15, Kuramoto-cho,

Tokushima-city, Tokushima 770-8503, Japan

e-mail: hinoue@genome.tokushima-u.ac.jp

N. Nakamura $\cdot$ T. Yoshikawa

Department of Endocrinology and Metabolism,

Graduate School of Medical Science,

Kyoto Prefectural University of Medicine, Kyoto, Japan

N. Yasui

Department of Orthopedics, Institute of Health Biosciences,

The University of Tokushima, Tokushima, Japan

H. Shiota

Department of Ophthalmology and Visual Neuroscience, Institute for Health Biosciences, The University of Tokushima,

Tokushima, Japan
These disease-associated SNPs were genetically indistinguishable because of the presence of strong LD, as found previously in Caucasians. For the KCNJ11 E23K variant, the most significant association was obtained under a dominant genetic model (OR 1.32, 95\% CI 1.09-1.60, $P=0.004)$. A meta-analysis of East Asian studies, comprising a total of 3,357 T2D patients (77.4\% Japanese) and 2,836 control subjects (77.8\% Japanese), confirmed the significant role of the KCNJ11 E23K variant in T2D susceptibility. Furthermore, we found evidence suggesting that the KCNJ11 E23K genotype is independently associated with higher blood-pressure levels.

Keywords Type 2 diabetes - KCNJ11 - ABCC8 . Genetic association study · Japanese population . Blood pressure

\section{Introduction}

The pancreatic $\beta$-cell $\mathrm{K}_{\mathrm{ATP}}$ channel plays a central role in glucose-stimulated insulin secretion from $\beta$-cells, and is composed of two essential subunits: the Kir6.2, a member of the inwardly rectifying potassium channel superfamily, and the sulfonylurea receptor 1 (SUR1) (Aguilar-Bryan et al. 1998; Seino and Miki 2003; Koster et al. 2005). It is widely accepted that common hypoglycemic sulfonylurea drugs bind to SUR1 and inhibit the channel activity in $\beta$ cells, leading to membrane depolarization and subsequent $\mathrm{Ca}^{2+}$ entry through voltage-dependent $\mathrm{Ca}^{2+}$ channels and insulin exocytosis (Gribble and Reimann 2003). Genetic evidence also supports the importance of $\beta$-cell $\mathrm{K}_{\mathrm{ATP}}$ channels in insulin secretion. Heterozygous mutations in either Kir6.2 or SUR1 cause familial persistent hyperinsulinemic hypoglycemia of infancy (PHHI; OMIM 256450), 
a rare genetic disorder due to inappropriate insulin secretion in the face of hypoglycemia (Gloyn et al. 2006). Recent studies further showed activating mutations in the Kir6.2 to be common causes of permanent neonatal diabetes (PNDM; OMIM 606176), which is characterized by persistent, insulin-requiring hyperglycemia occurring in the first 3 months of life (Hattersley and Ashcroft 2005).

Kir6.2 and SUR1 are encoded by the two adjacent chromosome $11 \mathrm{p} 15.1$ genes, KCNJ11 and ABCC8, respectively. These $\beta$-cell $\mathrm{K}_{\mathrm{ATP}}$ channel subunit genes are thus excellent candidates for a susceptibility gene involved in the common form of type 2 diabetes (T2D). In fact, genetic associations with variants in these genes have been reported in many studies, with conflicting results for the $A B C C 8$ variants and more consistent results for the $K C N J 11$ variants. Recently, large-scale association studies and metaanalyses have strongly indicated that the KCNJ11 E23K variant (a glutamate-to-lysine amino acid substitution at position 23) is associated with T2D in Caucasian populations (Hani et al. 1998; Nielsen et al. 2003; Gloyn et al. 2003; Florez et al. 2004). The effect size of having the susceptible K23 allele seems to be modest (odds ratio [OR] of approximately 1.2): however, because approximately $60 \%$ of the population carries at least one K23 allele, it is likely to have a substantial effect on population-attributable risk. Data from in vitro functional experiments has indicated that the K23 type of Kir6.2 alters $\mathrm{K}_{\mathrm{ATP}}$ function by inducing spontaneous over-activity and a subsequent increase in the threshold ATP concentration for insulin release (Schwanstecher et al. 2002a; Riedel et al. 2003). Consistently, in glucose-tolerant subjects, significant associations of the KCNJ11 K23 allele with reductions in insulin release during an oral glucose tolerance test (OGTT) have been reported (Nielsen et al. 2003; Florez et al. 2004).

While current data strongly support the hypothesis that SNPs in the KCNJ11-ABCC8 locus associate with the risk of T2D in Caucasian populations, and that the KCNJ11 E23K variant is likely to be responsible for this link, whether KCNJ11 E23K is the sole, or best, marker of T2D susceptibility at this locus remains to be established. This is because the strong linkage disequilibrium (LD) across the $K C N J 11$ gene, which extends into the $A B C C 8$ gene, leaves open the possibility that other variants (in either gene) might contribute to the associations observed (Florez et al. 2004; Koster et al. 2005). In addition, associations of SNPs in the KCNJ11-ABCC8 locus with T2D are poorly defined in non-Caucasian populations. The KCNJ11 E23K variant is reportedly less common in individuals of African descent (Florez et al. 2007), making it difficult to explore the impact of this variant on T2D susceptibility. In East Asian subjects, the KCNJ11 E23K variant appears to be fairly common, but only a few small or medium-scale association studies have been done, and the results were inconclusive
(Yamada et al. 2001; Liu et al. 2006; Yokoi et al. 2006; Koo et al. 2007). In the present study, to better clarify the contribution of genetic variants in the KCNJ11-ABCC8 gene region to T2D susceptibility in East Asian populations, we characterized the LD and haplotype structure of this locus, and conducted a case-control study in 1,802 unrelated Japanese individuals. In addition, we reviewed case-control studies of the KCNJ11 E23K variant that involved Japanese and Korean populations employing a meta-analysis, in which we confirmed the significant association of the KCNJ11 E23K variant with T2D in East Asian populations. Furthermore, we obtained evidence suggesting the KCNJ11 E23K genotype is possibly associated with blood pressure levels and thus susceptibility to the development of hypertension.

\section{Subjects and methods}

Study subjects

This study was conducted in accordance with the tenets of the Declaration of Helsinki. All subjects consented to participate in the process approved by the Ethics Committee for Human Genome/Gene Research at the University of Tokushima. The study population consisted of 909 unrelated Japanese patients with T2D (50.0\% females; average age at recruitment $63.9 \pm 9.6$ [SD] years; average age at T2D diagnosis $44.8 \pm 11.4$ years) and 893 control subjects (50.9\% females; average age at recruitment $37.5 \pm 11.8$ years). Subject recruitment and clinical characteristics were previously described in detail (Keshavarz et al. 2006; Tanahashi et al. 2006; see also Electronic supplementary material [ESM], Table 1). Briefly, subjects with T2D were recruited between January 2000 and December 2003 from two sources: (1) 349 T2D subjects who were referred to the Eye Clinic at the Tokushima University Hospital and screened for diabetic retinopathy; (2) $560 \mathrm{~T} 2 \mathrm{D}$ subjects who visited the outpatient clinic of the Kyoto Prefectural University Hospital and affiliated institutions. A detailed clinical questionnaire, including history of T2D and other metabolic diseases such as hypertension, family history, and detailed physical examination findings was obtained for each patient. The diagnosis of T2D was made either by the 1985 WHO criteria or by being treated with medication for diabetes. Hypertension was defined as mean systolic blood pressure $\geq 140 \mathrm{mmHg}$ and/or mean diastolic blood pressure $\geq 90 \mathrm{mmHg}$ and/or current or previous treatment with antihypertensive drugs. Mean arterial blood pressure (MBP) was calculated according to the formula: (systolic-diastolic) $/ 3$ + diastolic blood pressure. Control subjects consisted of two groups: (1) 372 unrelated healthy volunteers were employees of the University of Tokushima and 
related hospitals; (2) 521 Epstein-Barr virus transformed B-lymphoblast cell lines, derived from unrelated healthy volunteers and established by the Pharma SNP consortium (PSC), were obtained through the Japan Health Sciences Foundation (JHSF)/Health Science Research Resources Bank (HSRRB). Genomic DNAs were extracted either from peripheral blood leukocytes or Epstein-Barr virus immortalized B-lymphoblasts by standard techniques.

\section{Selection and genotyping of SNPs}

SNPs were selected from among various reports in the literature and public sources, such as the NCBI dbSNP (http://www.ncbi.nlm.nih.gov/SNP/), ABI (http:// www.appliedbiosystems.com) and HapMap (http://www. hapmap.org/) databases. In total, we selected 31 SNPs in the KCNJ11-ABCC8 locus (Table 1), based on the principles of equal density across genes, allelic frequencies, and SNP availability. All SNPs were genotyped using the TaqMan allelic discrimination assay with allele-specific fluorescent MGB probes, obtained from Applied Biosystems (Foster City, CA, USA). PCR was performed with a QuantiTect Probe PCR Master Mix (Qiagen, Valencia, CA, USA), with primer concentrations of $900 \mathrm{nM}$ and MGBprobe concentrations of $200 \mathrm{nM}$, in a total reaction volume of $4 \mu$ l. Five nanograms of genomic DNA were used as a template. After the reaction, assay plates were transferred to ABI PRISM 7900HT instruments (Applied Biosystems), and the fluorescence intensity in each well was read. Fluorescence data files from each plate were first analyzed by automated allele calling with the "Qaulity Value" setting of 95.0 (SDS2.1 software, Applied Biosystems), and were then independently reviewed by two experienced operators (Y.S. and H.I.). Individual genotypes that were ambiguous were designated as "undetermined", and SNPs with an "undetermined" call rate of $\geq 2 \%$ per assay were re-genotyped. Our high-throughput genotyping was performed satisfactorily and the overall genotyping success rate was $99.5 \%$.

\section{Statistical analysis}

The Hardy-Weinberg equilibrium (HWE) tests of SNPs were performed using SNPAlyze Pro software (Version 5.1; Dynacom, Yokohama, Japan), and were carried out for all SNP loci of patients and controls separately. Pair-wise LD coefficients, $\left|\mathrm{D}^{\prime}\right|$ and $r^{2}$ (Gabriel et al. 2002), were determined, and visualized with the GOLD program (available at http://www.sph.umich.edu/csg/abecasis/GOLD/docs/ldmax. html). Genotypic and allelic frequencies of the SNPs in the case and control groups were compared using a $\chi^{2}$ test. Analyses were also performed assuming dominant, co- dominant (additive) and recessive genetic models, and the crude odds ratios (ORs), their $95 \%$ confidence interval (CI) ranges and corresponding $P$ values were calculated using the Web-Assotest program (available at http://www. ekstroem.com/). Logistic regression was used to estimate both unadjusted and age-, gender- and body mass index (BMI)-adjusted ORs. Correction for multiple testing was performed using Storey's $q$ value method (Storey and Tibshirani 2003) employing the QVALUE program (available at http://faculty.washington.edu/jstorey/qvalue/), in which $P$ values were adjusted according to the experimental false discovery rate (FDR). If $q$ values $<0.05$ are taken as significant, there is a FDR of $5 \%$ among the significant associations. The SNP haplotypes and their frequencies were estimated by the maximum-likelihood method with an expectation-maximization (EM) algorithm using SNPAlyze Pro. Permutation $P$ values were calculated by comparing haplotype frequencies between cases and controls on the basis of 10,000 replications. Analysis of variance (ANOVA) or the unpaired two-tailed Student's $t$ test was performed to quantitatively compare clinical data among populations or genotypes. The significance level for statistical tests was set at 0.05 . Associations between blood pressure levels and the KCNJ11 E23K genotypes were evaluated by multiple logistic regression analysis, with blood pressure levels (systolic, diastolic or mean) being regarded as the dependent variable, and genotypes, T2D (presence or absence), age, gender $($ male $=0$; female $=1)$ and BMI as the independent variables. All data management and statistical computations were performed using SPSS for Windows (Version 12.0; SPSS Inc., Tokyo, Japan). Statistical power to detect an association was determined with the PS power and sample-size program (available at http://www.mc.vanderbilt.edu/prevmed/ps). The meta-analysis was performed employing the MantelHaenszel approach as a fixed-effects model test and the DerSimonian-Laird method as a random-effects model test, using StatsDirect statistical software (Version 2.6.2; StatsDirect Ltd., Sale, UK). Heterogeneity among studies was evaluated by means of Cochran's $Q$ test. Estimates of population-attributable risk (PAR) were calculated with the use of the formula PAR $=\operatorname{Pr}(\mathrm{OR}-1) /(1+\operatorname{Pr}(\mathrm{OR}-1))$, where $\mathrm{Pr}$ is the prevalence of risk genotypes in a population (Lillienfeld and Lillienfeld 1980).

\section{Results}

Selected SNPs and patterns of LD in the KCNJ11-ABCC8 gene locus

In the KCNJ11-ABCC8 gene locus, we selected a total of 31 SNPs, spanning a 211.3-kb genomic segment and 


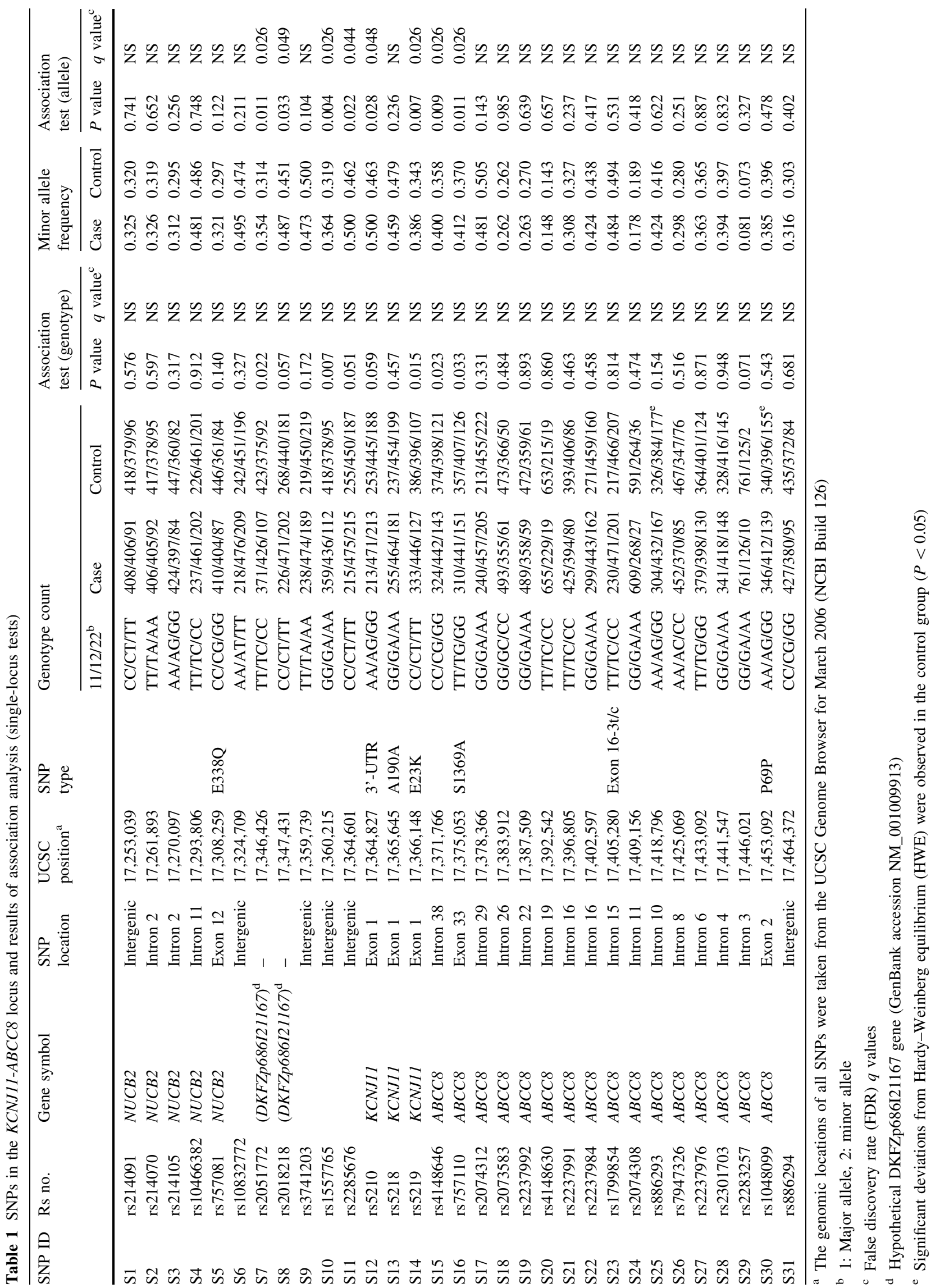


covering the KCNJ11 and $A B C C 8$ genes, as well as two neighboring genes: NUCB2 (Nucleobindin-2) and hypothetical DKFZp686121167. The SNP names, features and positions relative to the genes are given in Table 1 and Fig. 1a. Among these SNPs, six were located within exons and untranslated regions, and at least three of them had previously been reported to be associated with T2D in various populations: i.e., rs5219 (KCNJ11 E23K), rs757110 (ABCC8 S1369A) and rs1799854 (ABCC8 exon $16-3 \mathrm{t} / \mathrm{c})$. The average SNP spacing was $7.04 \pm 5.78 \mathrm{~kb}$ (median $5.58 \mathrm{~kb}$, min-max, 226-23,709 bp), and the average minor allele frequency (MAF) in control subjects was $0.36 \pm 0.11$ (min-max, 0.07-0.50). Among control individuals, the genotype distributions of two SNPs (rs886293 and rs1048099) deviated from our criteria for HWE $(P<0.01)$. However, we included these SNPs in further analyses, because we used a stringent genotypingscoring protocol to eliminate possible genotyping errors and found no discrepancies in duplicated genotyping, indicating that the deviations were probably due to chance.

Using the genotype data from 893 control individuals, we initially characterized the extent and pattern of LD of the KCNJ11-ABCC8 gene locus by the two LD measures, ID'I and $r^{2}$. The results revealed the KCNJI1-ABCC8 gene locus to be characterized by significant $L D$, and this was particularly evident with ID'|-based analysis (Fig. 1a, upper). There were at least eight regions of consistent and strong LD with low haplotype diversity (LD block), as defined by the standard criteria of Gabriel et al. (2002) (Fig. 1a, lower, LD blocks B1 to B8). Notably, LD blocks $\mathrm{B} 1$ to $\mathrm{B} 5$ comprise a large $125-\mathrm{kb}$ genomic region with moderately high LD, spanning from rs214091 to rs2074312, which includes all exons of the KCNJ11, DKFZp686I21167 and NUCB2 genes, and also contains the 3'-portion of the $A B C C 8$ gene. Other portions of the $A B C C 8$ gene could be broken down into at least three LD
Fig. 1 a Pairwise LD of 31

SNPs from the 211.3-kb

genomic region containing $K C N J 11, A B C C 8$ and

neighboring known genes, NUCB2 and DKFZp686I21167 (hypothetical gene). SNP numbers correspond to ID numbers in Table 1 . Colorcoded $\left|D^{\prime}\right|$ values for pairs of SNPs are plotted using the GOLD program, with scores of 1 (shown in red) and 0 (in dark blue). A physical and gene map of the genomic region is shown below. The exons and introns are represented as vertical and horizontal lines respectively. Following the haplotype block definition of Gabriel et al. (2002), there are eight distinct LD blocks (B1 to B8, shown as black lines) in this genomic region. b Allelic $P$ values (negative log scale) of 31 SNPs are plotted against genomic positions (obtained from the UCSC Genome Browser, March 2006 Human Genome Assembly, NCBI dbSNP Build 126 ), and a $P$ value of 0.05 is indicated with a red dashed line. SNPs previously reported to be associated with T2D, rs5219 (KCNJ11 E23K), rs757110 (ABCC8 S1369A) and rs1799854 (ABCC8 exon 16-3t/ c), are indicated with black arrows a

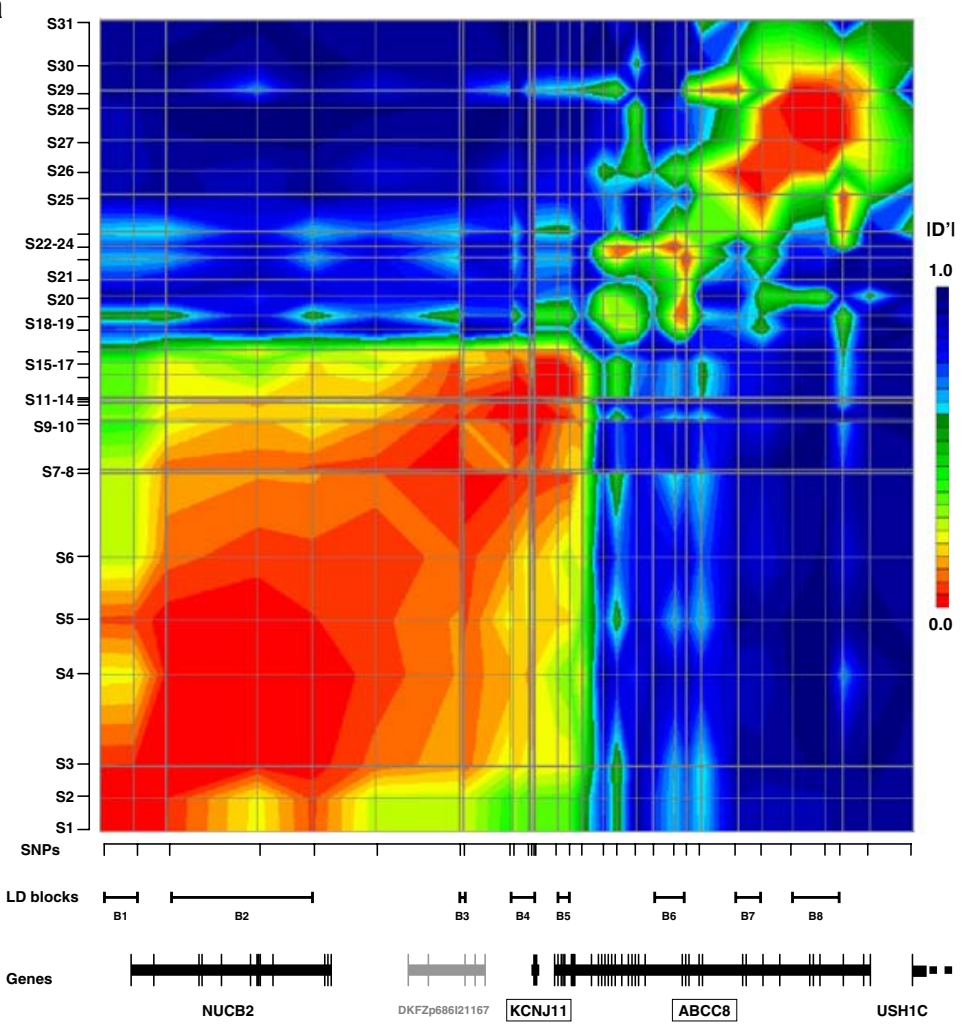

b

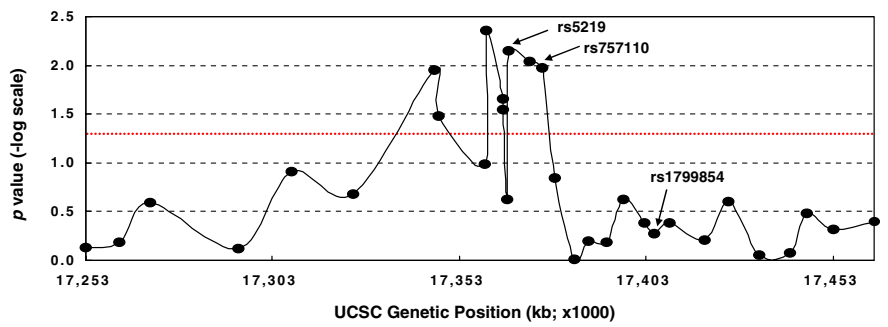


blocks (B6 to B8). We observed essentially the same pattern when LD was assessed by the more stringent measure of $r^{2}$, which also accounts for differences in allele frequencies (ESM, Fig. 1a). Overall, we noted that the LD pattern of the KCNJ11-ABCC8 gene region in the Japanese samples appeared to be consistent with the results of Florez et al. (2004), who reported that the KCNJ11 gene and portions of the $A B C C 8$ gene were contained in an at least 75-kb LD block showing high LD in a Caucasian population.

Association between SNPs in the KCNJ11-ABCC8 locus and $\mathrm{T} 2 \mathrm{D}$

We genotyped all selected SNPs in the KCNJ11-ABCC8 locus in 909 Japanese patients with T2D and 893 control subjects. The results of the allelic and genotypic association study are summarized in Table 1. Eight SNPs (rs2051772, rs2018218, rs1557765, rs2285676, rs5210, rs5219, rs4148646 and rs757110) showed a nominally significant allelic or genotypic association $(0.004<P<0.033)$. These included the previously reported T2D-associated SNPs, rs5219 (KCNJ11 E23K) and rs757110 (ABCC8 S1369A), but not rs1799854 (ABCC8 exon $16-3 \mathrm{t} / \mathrm{c})$. The observed associations were significant even after a QVALUE correction test (FDR $q<0.05$ ), though significance was lost with conservative Bonferroni correction (Bonferroni significance threshold $P=0.0016[0.05 / 31$ tests $]$ ). Figure $1 \mathrm{~b}$ shows a graphic representation of the case-control test results for allelic associations with $\mathrm{T} 2 \mathrm{D}$, plotted against their relative genomic positions and LD blocks. The T2Dassociated SNPs were found to be concentrated around the $K C N J 11$ gene, but also resided within the nearby $A B C C 8$ and DKFZp686121167 genes. It can also be seen that these SNPs are located at the centromeric end of the aforementioned 125-kb genomic region with high LD (Fig. 1a).

Because the eight T2D-associated SNPs were in significant LD with each other $\left(0.47<r^{2}<1.00\right.$; ESM, Fig. 1b), and their significance levels appeared to be similar, we assumed that a haplotype-based association study might be useful for identifying the actual causative variant(s), not necessarily genotyped in the present study. The putative T2D-associated SNPs were found to be included in three adjacent, closely related $\left(\mathrm{D}^{\prime}>0.9\right)$ yet distinct $\mathrm{LD}$ blocks, B3 to B5 (Figs. 1b, 2a). When the haplotype association test was performed on each LD block, three atrisk haplotypes (B3:11, B4:011101, and B5:11; Fig. 2a, table) were found to be in significant excess in T2D patients, relative to control subjects. A closer look revealed that these three at-risk haplotypes were most frequent in combinations across block boundaries, and carried variant alleles for both rs5219 (KCNJ11 E23K) and rs757110
(ABCC8 S1369A). In addition, the significance levels for each at-risk haplotype were similar to those of single-locus tests. These results together indicated that there was no significant improvement in identifying the specific contribution of individual SNPs by our LD block-structured haplotype association testing. We have tested alternative haplotype block definitions as well as different SNP combinations for the haplotype association test, but the results were essentially the same (data not shown). Furthermore, to specify the relationship between the rs5219 (KCNJ11 E23K) and rs757110 (ABCC8 S1369A), we conducted a two-SNP haplotype (combination) analysis (Fig. 2b), and the most significant association was observed for haplotype 11 , which again carried both variant alleles. Haplotype 01 consisted of the wild-type allele of KCNJ11 E23K and the variant allele of $A B C C 8$ S1369A, potentially making individual effects of these variants on the disease susceptibility distinguishable. As haplotype 01 showed no significant association with disease status, a negative role of the ABCC8 S1369A variant was suggested: however, these results cannot be considered conclusive, because the analysis was apparently underpowered and lacked detection power due to low haplotype frequency (approximately $2.5 \%$ ) and the small sample size of the current study.

Associations of KCNJ11 E23K variant with T2D and blood pressure levels

Because our T2D-associated SNPs, including rs5219 $(K C N J 11 \mathrm{E} 23 \mathrm{~K})$ and $\operatorname{rs} 757110$ (ABCC8 S1369A), were found to be genetically and statistically indistinguishable, and, in Caucasian populations, the most consistent evidence for association with T2D has been obtained with KCNJ11 E23K, we decided to focus our subsequent investigation on this variant. When we tested the association of KCNJ11 E23K with T2D under a range of genetic models in our population, the most significant results were obtained using a dominant model (KK/EK vs EE; OR 1.32, 95\% CI 1.09-1.60; $P=0.004$, age-, sex- and BMI-adjusted OR $1.46,95 \%$ CI $1.05-2.04, P=0.023$, Table 2), while the association was weaker and insignificant under a recessive model (KK vs EK/EE; OR 1.19, 95\% CI 0.90-1.37; $P=0.21$, age-, sex- and BMI-adjusted OR $1.37,95 \%$ CI $0.86-2.19, P=0.19)$. These results were in contrast to those from the Caucasian studies (Gloyn et al. 2003; Florez et al. 2004), showing stronger associations of the K23 allele under a recessive model.

Table 3 shows the clinical characteristics of each of the KCNJ11 E23K genotypes of T2D and the control subjects. Among individuals with T2D, one-way ANOVA showed that the respective systolic and mean blood pressure values differed significantly across the E23K genotypes $(P=0.027$ 
a

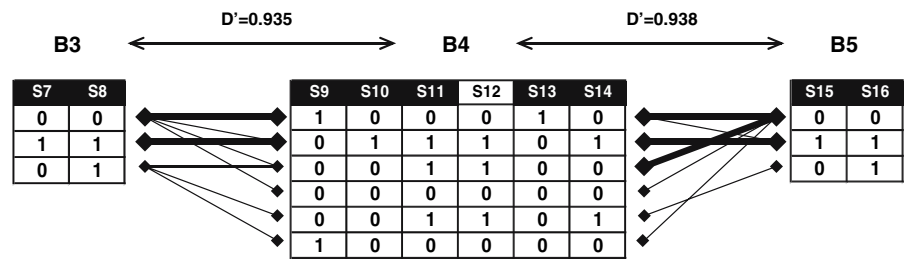

\begin{tabular}{ccccccc}
\hline & \multirow{2}{*}{ Haplotype } & \multicolumn{3}{c}{ Frequency } & \multirow{2}{*}{$P$ value } & \multirow{2}{*}{ Permutation $P$ value } \\
\cline { 2 - 4 } & & T2D & Control & & 0.023 \\
\hline \multirow{3}{*}{ B3 } & 00 & 0.512 & 0.548 & & 0.030 & 0.012 \\
& 11 & 0.354 & 0.314 & & 0.012 & 0.77 \\
\hline \multirow{6}{*}{ B4 } & 01 & 0.135 & 0.138 & 0.77 & 0.16 \\
& 100010 & 0.454 & 0.477 & 0.18 & 0.0053 \\
& 011101 & 0.361 & 0.318 & & 0.0061 & 0.59 \\
& 001100 & 0.112 & 0.118 & & 0.59 & 0.07 \\
& 000000 & 0.024 & 0.034 & & 0.06 & 0.79 \\
& 001101 & 0.029 & 0.028 & & 0.80 & 0.24 \\
\hline \multirow{3}{*}{ B5 } & 100000 & 0.020 & 0.026 & 0.24 & 0.0090 \\
& 00 & 0.589 & 0.631 & 0.0091 & 0.0086 \\
& 11 & 0.400 & 0.358 & 0.0094 & 0.88 \\
\hline
\end{tabular}

b

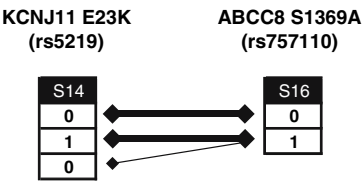

\begin{tabular}{|c|c|c|c|c|c|}
\hline & \multirow{2}{*}{ Haplotype } & \multicolumn{2}{|c|}{ Frequency } & \multirow{2}{*}{$P$ value } & \multirow{2}{*}{ Permutation $P$ value } \\
\hline & & T2D & Control & & \\
\hline \multirow{3}{*}{ S14-S16 } & 00 & 0.588 & 0.631 & 0.0088 & 0.010 \\
\hline & 11 & 0.386 & 0.344 & 0.0092 & 0.0090 \\
\hline & 01 & 0.026 & 0.025 & 0.88 & 0.85 \\
\hline
\end{tabular}

Fig. 2 a Haplotype structure and diversity for LD blocks, B3 to B5, containing putative T2D-associated SNPs. SNP numbers corresponding to Table 1 are listed above each column of alleles, and the haplotype-tagging SNPs are indicated by white letters with a black background. SNPs S7 and S8 (LD block B3), S10, S11, S12 and S14 (B4), S15 and S16 (B5) showed significant single-locus allelic associations with T2D. LD blocks were specified according to the definition of Gabriel et al. (2002), and haplotype frequencies were estimated using the expectation-maximization algorithm implemented in SNPAlyze Pro software. In each LD block, the compositions of SNP haplotypes are shown in descending order of estimated frequency $(O$ major allele; 1 minor allele). Haplotypes in adjacent blocks are connected by a thick line, if they occur together with a frequency $>10 \%$, and by a thin line with a frequency $>1 \%$. The inter-block $\mathrm{D}^{\prime}$ scores between $\mathrm{B} 3$ and $\mathrm{B} 4$, or $\mathrm{B} 4$ and $\mathrm{B} 5$, are shown above. The lower table shows the estimated haplotype frequencies and results of association testing with T2D. For the correction of multiple comparisons, the permutation test was performed (10,000 iterations). b Association of estimated two-SNP (S14: rs5219-S16: rs757110) haplotype frequencies with T2D. Note that haplotype 01 consisted of the wild-type allele of $K C N J 11 \mathrm{E} 23 \mathrm{~K}$ and the variant allele of $A B C C 8 \mathrm{~S} 1369 \mathrm{~A}$, making individual effects on disease-susceptibility potentially distinguishable

Table 2 Odds ratios for the associations between KCNJ11 E23K (rs5910) and type 2 diabetes (T2D)

\begin{tabular}{|c|c|c|c|c|c|}
\hline \multirow[t]{2}{*}{ KCNJ11 E23K (rs5910) } & \multicolumn{2}{|l|}{ Frequency } & \multicolumn{3}{|l|}{ OR $(95 \% \mathrm{CI})$} \\
\hline & $\mathrm{T} 2 \mathrm{D}(\%)$ & Control (\%) & Relative OR & 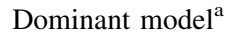 & Recessive model ${ }^{\mathrm{a}}$ \\
\hline EE & $333(36.8)$ & $386(43.4)$ & 1.00 & $1.32(1.09-1.60)^{\mathrm{b}}$ & $1.19(0.90-1.57)$ \\
\hline EK & $446(49.2)$ & $396(44.5)$ & $1.31(1.07-1.59)$ & & \\
\hline KK & $127(14.0)$ & $107(12.0)$ & $1.38(1.02-1.85)$ & & \\
\hline
\end{tabular}

OR odds ratio, $C I 95 \%$ confidence interval

a The dominant model is $\mathrm{KK} / \mathrm{EK}$ versus $\mathrm{EE}$, the recessive model $\mathrm{KK}$ versus $\mathrm{EK} / \mathrm{EE}$

${ }^{\mathrm{b}}$ Age, sex and BMI-adjusted OR $1.46(95 \%$ CI 1.05-2.04, $P$ value $=0.023)$

and 0.011 respectively); i.e. systolic and mean blood pressure levels were higher in subjects with the KK genotype $(144.5 \pm 21.7$ and $101.3 \pm 14.3 \mathrm{mmHg}$ respectively) than in those with the EK $(141.1 \pm 21.0$ and $97.8 \pm 12.6 \mathrm{mmHg})$ and EE genotypes $(135.8 \pm 18.5$ and $94.8 \pm 10.7 \mathrm{mmHg})$. The same tendency was observed for diastolic blood 
pressure in the T2D group, as well as for each of the blood pressure values in the control group, though the differences were not statistically significant. Pooling the T2D with control subjects made differences in systolic, diastolic and mean blood pressure levels more statistically significant ( $P=0.001$ for systolic, $P=0.006$ for diastolic and $P<0.001$ for mean blood pressures; Table 3). Age and BMI did not differ significantly between genotypes. To assess the independent contribution of these variables to blood pressure levels, we performed multiple regression analyses, and found the KCNJ11 E23K genotype to be independently related to blood pressure levels $(P<0.05$; Table 4). In addition, there was an indication that T2D subjects with the KCNJ11 KK genotype had more severe hyperglycemia than subjects with EK or EE, as the lifetime maximum plasma glucose and maximum HbA1c levels were significantly higher $(P=0.004$ and $P<0.001$ respectively; Table 3). On the other hand, there were no significant differences among the three genotype groups in either current insulin use (\%) or complication status (nephropathy and proliferative retinopathy).

Because we observed an association between KCNJ11 E23K genotypes and blood pressure levels, we compared allelic and genotypic frequencies between hypertensive and normotensive individuals. In our population, there were 546 hypertensive (96.8\% with T2D) and 687 normotensive (52.8\% with T2D) subjects. The KCNJ11 E23K genotype distribution in patients with hypertension $(\mathrm{EE}=36.1 \%$ $[n=203], \mathrm{EK}=50.7 \%[n=285], \mathrm{KK}=13.2 \%[n=74])$ did not differ from that in normotensive subjects $(\mathrm{EE}=$ $42.5 \%[n=290], \mathrm{EK}=44.9 \% \quad[n=307], \mathrm{KK}=12.6 \%$ $[n=86] ; P=0.069)$. There was a weak but statistically significant association under a dominant genetic model (KK/EK vs EE; OR 1.31, 95\% CI 1.04-1.65, $P=0.021$ ), though the associations disappeared after adjustment for sex, age and BMI (OR 1.18, 95\% CI 0.89-1.55, $P=0.25$ ). However, we speculate that the association might be due to an indirect effect resulting from the higher prevalence of $\mathrm{T} 2 \mathrm{D}$ in our hypertension group, attributable in part to the nature of the current study design. In this regard, we were unable to assess disease association in a group of non-T2D, hypertensive subjects as compared with non-T2D, normotensive control subjects, because only a few subjects (about $5 \%$ ) were hypertensive in our non-T2D control group.

\section{Meta-analysis of East Asian-population studies}

We performed an OR meta-analysis of East Asian studies on the KCNJ11 E23K variant. We found four original published association studies dealing with this variant in T2D (Yamada et al. 2001; Yokoi et al. 2006; Liu et al. 2006; Koo et al. 2007): however, unfortunately, the data available for the Han Chinese population were not sufficiently detailed to be included in the present analysis. Adding the present data to those available from the other three studies (Yamada et al. 2001; Yokoi et al. 2006; Koo et al. 2007) allowed a total of 3,357 subjects with T2D (77.4\% Japanese) and 2,836 control individuals (77.8\% Japanese) to be analyzed for disease associations. We assumed a dominant genetic model of inheritance $(\mathrm{KK} / \mathrm{EK}$ vs EE), because the most significant association results were obtained in our Japanese population using this genetic model. As shown in Fig. 3, our meta-analyses based on all studies or only those on Japanese populations produced significant associations: fixed-effects OR 1.18, 95\% CI 1.05-1.33, $P=0.0048$ and random-effects OR $1.20,95 \%$ CI $1.03-1.40, P=0.019$ among Japanese only, and fixedeffects OR 1.23, 95\% CI 1.11-1.37, $P<0.0001$ and random-effects OR 1.26, 95\% CI 1.09-1.46, $P=0.0019$ for all studies. There was no significant heterogeneity (Cochran's $Q$ test $P=0.25$ and 0.17 for Japanese-only and all studies respectively) and no evidence of publication bias (Eggers test $P=0.46$ for all studies). To quantify the impact of $K C N J 11 \mathrm{E} 23 \mathrm{~K}$, we calculated the estimated population attributable risk, which suggested that, under a dominant model, the K23 allele might contribute to $9.13 \%$ of $\mathrm{T} 2 \mathrm{D}$ in the Japanese population and $10.7 \%$ in all East Asian populations.

\section{Discussion}

In our original Japanese population, we obtained significant evidence for an association between genetic variants in the KCNJ11-ABCC8 locus, including KCNJ11 E23K and $A B C C 8$ S1369A, and T2D. It should be mentioned that one limitation of this study is that our controls were not matched for age. With respect to age at the time of the interview and subsequent blood sampling, the control subjects were significantly younger than the case subjects $(37.5 \pm 11.8$ and $63.9 \pm 9.6$ years respectively, $P<0.001)$. Owing to the late onset of T2D, it is possible that at least some of the controls who were included in this study will develop T2D in the future. This potential misclassification in the control group due to the inclusion of undiagnosed cases, as well as inappropriate case definition, would bias and weaken any association between the KCNJ11-ABCC8 locus and T2D. However, the average age at T2D diagnosis in the patients was $44.8 \pm 11.4$ years, rather similar to the mean age of controls, although the difference was still statistically significant $(P<0.001)$. In addition, our controls were extensively interviewed about their health, and reported at least without strong family history of T2D. Therefore, they would have only a low risk of developing T2D and the overall population incidence is estimated to be well below 


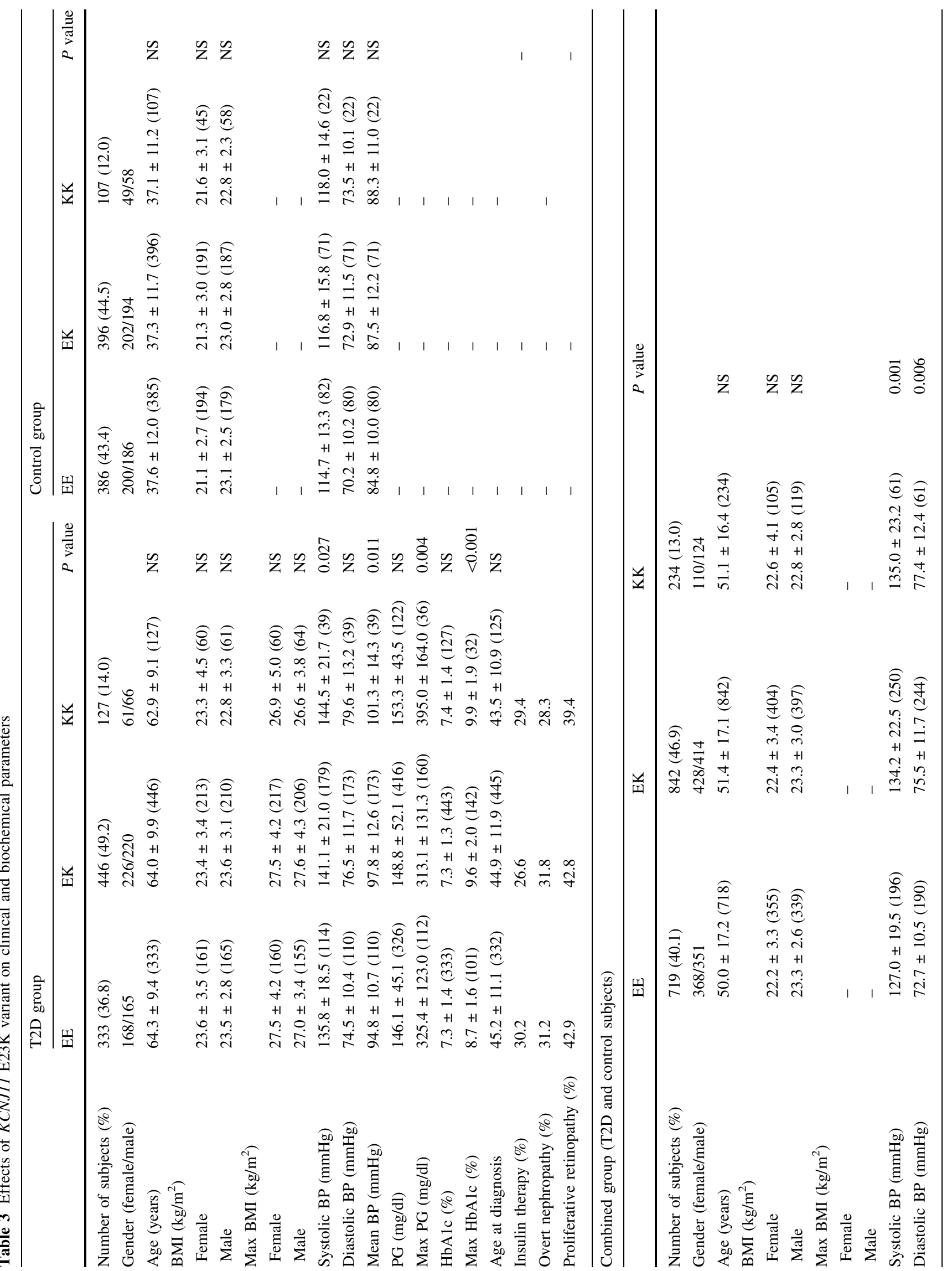




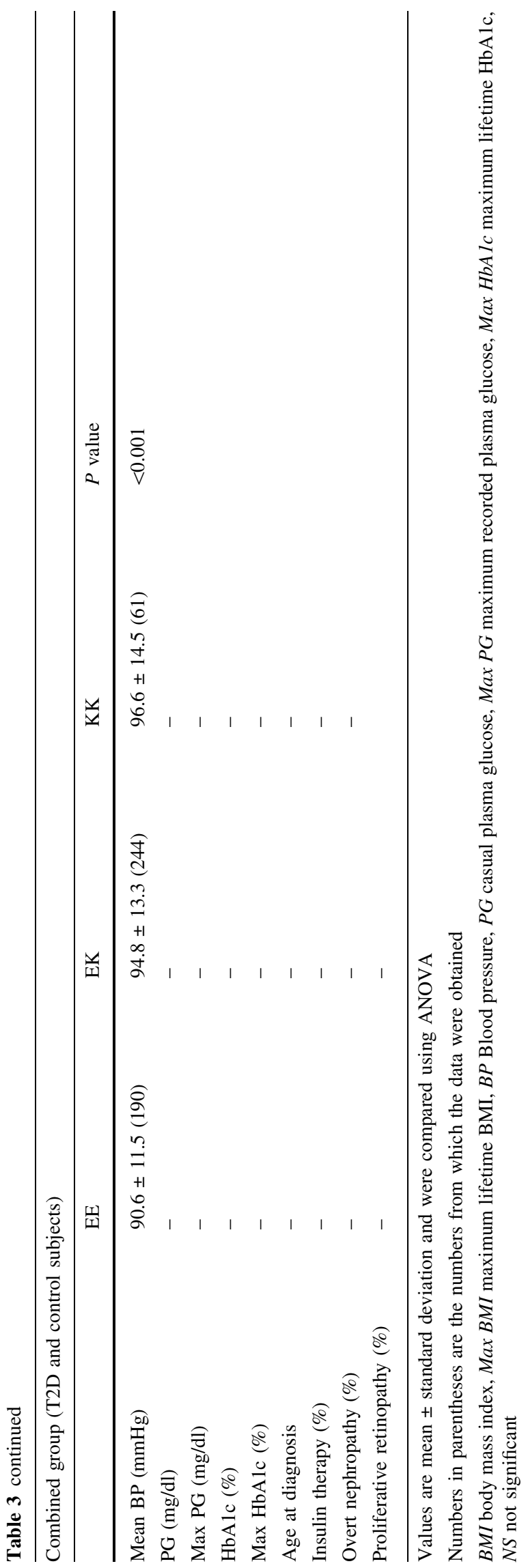

Table 4 Results of multiple regression analysis

\begin{tabular}{|c|c|c|c|c|}
\hline $\begin{array}{l}\text { Dependent } \\
\text { variable }\end{array}$ & Independent variables & Stand beta & $t$ & $P$ value \\
\hline \multirow[t]{6}{*}{ Systolic BP } & (Constant) & & 12.675 & $<0.001$ \\
\hline & $\begin{array}{c}\mathrm{T} 2 \mathrm{D} \text { (presence } \\
\text { or absence) }\end{array}$ & 0.281 & 5.044 & $<0.001$ \\
\hline & Age & 0.225 & 4.107 & $<0.001$ \\
\hline & BMI & 0.166 & 4.359 & $<0.001$ \\
\hline & $\begin{array}{l}\text { Gender }(\text { male }=0 \\
\text { female }=1)\end{array}$ & -0.103 & -2.689 & 0.007 \\
\hline & $\begin{array}{l}K C N J 11 \text { E23K } \\
\text { genotype }\end{array}$ & 0.091 & 2.445 & 0.015 \\
\hline \multirow[t]{4}{*}{ Diastolic BP } & (Constant) & & 14.976 & $<0.001$ \\
\hline & BMI & 0.264 & 6.155 & $<0.001$ \\
\hline & Age & -0.180 & -4.173 & $<0.001$ \\
\hline & $\begin{array}{l}\text { KCNJ11 E23K } \\
\text { genotype }\end{array}$ & 0.090 & 2.084 & 0.038 \\
\hline \multirow[t]{6}{*}{ Mean BP } & (Constant) & & 15.542 & $<0.001$ \\
\hline & BMI & 0.242 & 5.934 & $<0.001$ \\
\hline & $\begin{array}{l}\text { T2D (presence } \\
\text { or absence) }\end{array}$ & 0.202 & 3.359 & $<0.001$ \\
\hline & $\begin{array}{l}\text { Gender }(\text { male }=0 \\
\quad \text { female }=1)\end{array}$ & -0.154 & -3.745 & $<0.001$ \\
\hline & Age & 0.121 & 2.040 & 0.042 \\
\hline & $\begin{array}{l}\text { KCNJ11 E23K } \\
\text { genotype }\end{array}$ & 0.104 & 2.600 & 0.010 \\
\hline
\end{tabular}

Stand beta standardized beta-coefficient, $B P$ blood pressure, $T 2 D$ type 2 diabetes, $B M I$ body mass index

$10 \%$. Thus, the sample size of our association study should still provide adequate power to detect an association. On the other hand, due to the cross-sectional, population-based nature of this study, our findings need to be further confirmed by other independent populations and/or familybased tests of association. In the first case, the use of populations collected for a large prospective cohort study would be one obvious approach.

The present study represents, to our knowledge, the first positive results for the KCNJ11 E23K variant in the Japanese population. However, we noted that, even in the two previous studies on Japanese not obtaining significant results (Yamada et al. 2001; Yokoi et al. 2006), directions of the associations were consistent with our observations (Fig. 3), and the results of meta-analysis appear to support the notion that KCNJ11 E23K is associated with an increased T2D risk in East Asian populations, with an OR similar to those for Caucasian populations (Gloyn et al. 2003; Florez et al. 2004). Obviously, larger population sizes are required to confirm the small-to-moderate effects of KCNJ11 E23K variants on T2D risk in East Asian populations. In this regard, our power analysis showed that the present meta-analysis had more than $97.5 \%$ statistical power to detect significant associations at a 
Fig. 3 Meta-analysis of the association between $K C N J 11$ E23K and T2D in East Asian populations. Four studies, including this investigation, were included (Yamada et al. 2001; Yokoi et al. 2006; Koo et al. 2007). Black squares indicate the individual odds ratios (ORs) of the studies, with the size of each square being inversely proportional to its variance, and horizontal lines representing the $95 \%$ confidence interval (95\% CI). The pooled ORs and their $95 \%$ CI, calculated using fixed effects and random effects approaches, are indicated by the unshaded diamonds

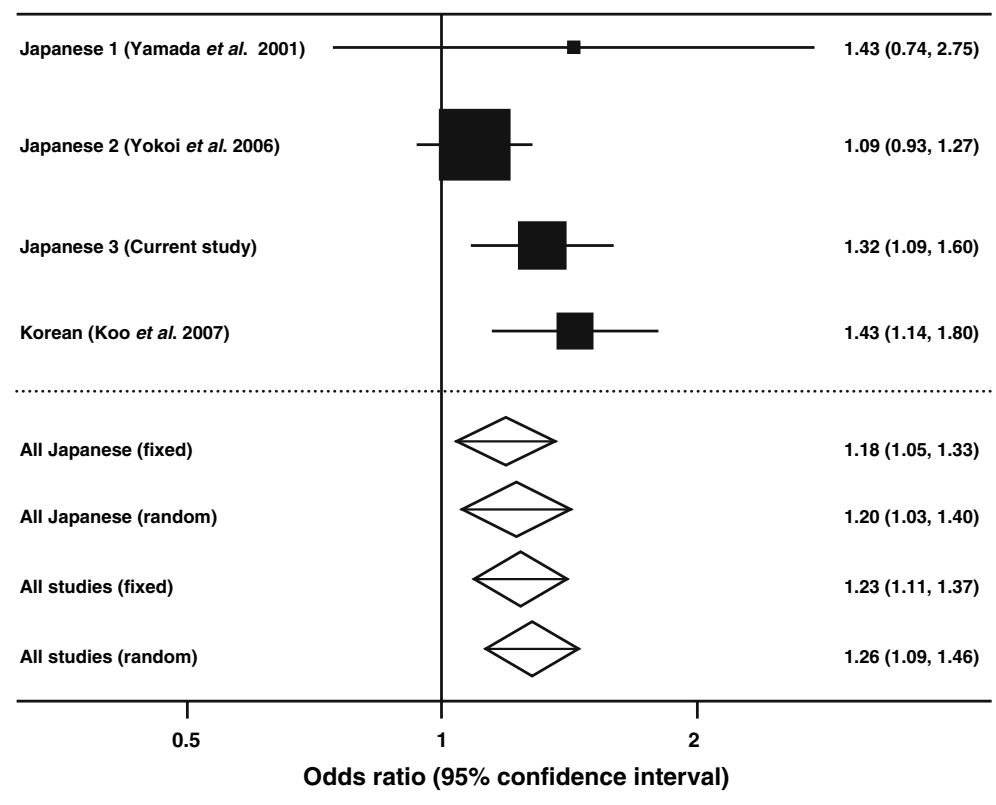

significance level of 0.05 , which further strengthens our conclusions.

The KCNJ11 E23K variant results in substitution of a highly conserved glutamate (E) residue with lysine (K) and a subsequent negative-to-positive shift in residue charge in the N-terminal cytosolic tail of the protein (Riedel et al. 2005). Previous functional reconstitution experiments indicated that expression of the KCNJ11 E23K variant results in altered $\mathrm{K}_{\mathrm{ATP}}$ channel activity, with reduced sensitivity to intracellular ATP, either by reducing ATP affinity or increasing intrinsic open-state stability, as well as enhanced MgADP and long-chain acyl-CoA esters (LCCoAs) stimulations (Schwanstecher and Schwanstecher 2002; Schwanstecher et al. 2002a, b; Riedel et al. 2003). Consequently, it has been postulated that these small changes in the electrical activity of pancreatic $\beta$-cells may affect insulin secretion. On the other hand, in Caucasian populations, it has been shown that KCNJ11 E23K has a strong allelic association with the $A B C C 8 \mathrm{~S} 1369 \mathrm{~A}$ variant, raising the possibility that this SUR1 variant may also influence altered channel activity, and that KCNJ11 E23K alone may not entirely account for the reported genetic association with T2D (Florez et al. 2004). In our Japanese population, as in Caucasians, we observed a high level of LD $\left(r^{2}>0.8\right)$ between the E23K and S1369A variants, making it difficult to distinguish their individual genetic effects. Due to this limitation, determination of whether the $A B C C 8$ S1369A variant, alone or in combination with $K C N J 11$ E23K, can account for altered $\beta$-cell $\mathrm{K}_{\mathrm{ATP}}$ channel activity awaits further genetic and biochemical studies. It is also noteworthy that there is one additional gene, DKFZp686121167, annotated as a hypothetical gene with no additional information in the database. As the
DKFZp686121167 gene is located approximately $8 \mathrm{~kb}$ downstream from $K C N J 11$ (Fig. 1a), and therefore may also be involved in the T2D-susceptibility associated with this locus, future investigations and experimental verifications of this hypothetical gene will be needed.

Interestingly, in this study, we observed the KCNJII E23K genotypes to have a significant influence on blood pressure levels. In the T2D group, carriers of the K23 allele had significantly higher blood pressures than subjects homozygous for the E23 allele. The same tendency was observed in the control group without T2D but did not reach statistical significance, while pooling of data from T2D and control subjects made differences more statistically significant. It should be mentioned, however, that the present investigation was designed to be a retrospective case-control study aimed at evaluating associations between genetic variations and the risk of T2D. Therefore, results from the secondary measures should be interpreted with appropriate caution, and require confirmation in future studies. On the other hand, supporting our findings, Koo et al. (2007) recently reported an association between the KCNJ11 SNPs including E23K and hypertension in their Korean population. Furthermore, Kokubo et al. (2006) investigated the association between 61 non-synonymous SNPs of candidate genes and blood pressure variations in a general population in Japan, and observed a moderate but significant blood pressure change in association with the $K C N J 11$ E23K genotypes, although the direction of association in this cohort seemed to be opposite that seen in the present study. Interestingly, the relationship between the KCNJ11 E23K genotype and blood pressure phenotypes has not previously been described in other racial/ethnic groups, suggesting that such an effect of the E23K 
genotype on blood pressure changes may be specific to East Asian populations.

Currently, the mechanism by which the KCNJ11 E23K variant may influence blood pressure levels remains unclear and requires further investigation. This association may merely be a secondary result of $\mathrm{T} 2 \mathrm{D}$ or disease severity, given the intimate relationship between T2D and hypertension. However, it is tempting to speculate that the increased activity of $\mathrm{K}_{\mathrm{ATP}}$ channels, as observed in the presence of the KCNJ11 E23K variant, has a direct impact on the cardiovascular system, and therefore is involved in susceptibility to hypertension. In addition to pancreatic islet cells, $\mathrm{K}_{\mathrm{ATP}}$ channel activities are found in many other cell types, including cardiac, smooth and skeletal muscle and neuronal cells, with cell-type specific combinations of channel subunits (Seino and Miki 2003): Kir6.2/SUR1 (neuronal and $\beta$-cell type), Kir6.2/SUR2A (cardiac and skeletal muscle type) and Kir6.1/SUR2B (smooth muscle type). Since activation of cardiac $\mathrm{K}_{\mathrm{ATP}}$ channels has been implicated in the regulation of electrical activity and muscle contractility as well as stress-adaptation (Zingman et al. 2002; Kane et al. 2005), the increased activity of $\mathrm{K}_{\text {ATP }}$ channels with the K23 allele may result in exaggerated responses to environmental triggers, which would in turn raise blood pressure. The vascular smooth muscle cell $\mathrm{K}_{\text {ATP }}\left(\mathrm{VSM}-\mathrm{K}_{\mathrm{ATP}}\right)$ channel is likely to be important in the control of vascular tone, and therefore of blood pressure (Brayden 2002). The subunit compositions of VSM-K $\mathrm{K}_{\text {ATP }}$ have not been determined definitively, though there is good evidence that a channel comprising the Kir6.1 and SUR2B plays important roles, at least in rodents (Seino and Miki 2003). The KCNJ11 E23K variant is thus unlikely to specifically affect the function of VSM-K $\mathrm{K}_{\mathrm{ATP}}$. Instead, the KCNJ11 E23K variant may alter $\mathrm{K}_{\mathrm{ATP}}$ channel activities in the central nervous system, and modulate the sympathetic nervous system, activation of which is thought to be important for the initiation and maintenance of high blood pressure and to contribute to hypertension. It is also possible that dysregulated secretion of hormones other than insulin, caused by the E23K variant, may affect control of blood pressure, since $\mathrm{K}_{\mathrm{ATP}}$ channels have been implicated in the secretion of many hormones, including glucagon and GLP-1 (Miki et al. 2001; Reimann and Gribble 2002), which play important roles in regulating water and electrolyte homeostasis and thereby affect blood pressure.

In summary, our association study in an independent Japanese population and a meta-analysis of East Asian studies confirm the genetic association between SNPs in the KCNJ11-ABCC8 locus, including KCNJ11 E23K and $A B C C 8$ S1369A, and T2D. Our findings, together with reported results in Caucasians, indicate the KCNJ11$A B C C 8$ locus to have a genuine association with $\mathrm{T} 2 \mathrm{D}$ across multiple racial/ethnic groups. We also obtained evidence suggesting an association between the KCNJ11 E23K variant and blood pressure. These results further support the notion that $\mathrm{K}_{\mathrm{ATP}}$ channel genes are involved in a broad variety of cardiovascular and metabolic phenotypes.

Acknowledgments We would like to thank our patients and volunteer blood donors for participating in this study. We also thank the many past and present members of our research group for helpful discussions. This study was partially funded by grants from the Ministry of Education, Science and Technology (Knowledge Cluster Initiative).

\section{References}

Aguilar-Bryan L, Clement JP, Gonzalez G, Kunjilwar K, Babenko A, Bryan J (1998) Toward understanding the assembly and structure of KATP channels. Physiol Rev 78:227-245

Brayden JE (2002) Functional roles of KATP channels in vascular smooth muscle. Clin Exp Pharmacol Physiol 29:312-316

Florez JC, Burtt N, de Bakker PI, Almgren P, Tuomi T, Holmkvist J, Gaudet D, Hudson TJ, Schaffner SF, Daly MJ, Hirschhorn JN, Groop L, Altshuler D (2004) Haplotype structure and genotypephenotype correlations of the sulfonylurea receptor and the islet ATP-sensitive potassium channel gene region. Diabetes 53:1360-1368

Florez JC, Jablonski KA, Kahn SE, Franks PW, Dabelea D, Hamman RF, Knowler WC, Nathan DM, Altshuler D (2007) Type 2 diabetes-associated missense polymorphisms KCNJ11 E23K and ABCC8 A1369S influence progression to diabetes and response to interventions in the Diabetes Prevention Program. Diabetes $56: 531-536$

Gabriel SB, Schaffner SF, Nguyen H, Moore JM, Roy J, Blumenstiel B, Higgins J, DeFelice M, Lochner A, Faggart M, Liu-Cordero SN, Rotimi C, Adeyemo A, Cooper R, Ward R, Lander ES, Daly MJ, Altshuler D (2002) The structure of haplotype blocks in the human genome. Science 296:2225-2229

Gloyn AL, Siddiqui J, Ellard S (2006) Mutations in the genes encoding the pancreatic beta-cell KATP channel subunits Kir6.2 (KCNJ11) and SUR1 (ABCC8) in diabetes mellitus and hyperinsulinism. Hum Mutat 27:220-231

Gloyn AL, Weedon MN, Owen KR, Turner MJ, Knight BA, Hitman G, Walker M, Levy JC, Sampson M, Halford S, McCarthy MI, Hattersley AT, Frayling TM (2003) Large-scale association studies of variants in genes encoding the pancreatic beta-cell KATP channel subunits Kir6.2 (KCNJ11) and SUR1 (ABCC8) confirm that the KCNJ11 E23K variant is associated with type 2 diabetes. Diabetes 52:568-572

Gribble FM, Reimann F (2003) Sulphonylurea action revisited: the post-cloning era. Diabetologia 46:875-891

Hani EH, Boutin P, Durand E, Inoue H, Permutt MA, Velho G, Froguel P (1998) Missense mutations in the pancreatic islet beta cell inwardly rectifying $\mathrm{K}+$ channel gene (KIR6.2/BIR): a metaanalysis suggests a role in the polygenic basis of type II diabetes mellitus in Caucasians. Diabetologia 41:1511-1515

Hattersley AT, Ashcroft FM (2005) Activating mutations in Kir6.2 and neonatal diabetes: new clinical syndromes, new scientific insights, and new therapy. Diabetes 54:2503-2513

Kane GC, Liu XK, Yamada S, Olson TM, Terzic A (2005) Cardiac KATP channels in health and disease. J Mol Cell Cardiol 38:937-943

Keshavarz P, Inoue H, Sakamoto Y, Kunika K, Tanahashi T, Nakamura N, Yoshikawa T, Yasui N, Shiota H, Itakura M (2006) No evidence for association of the ENPP1 (PC-1) K121Q variant 
with risk of type 2 diabetes in a Japanese population. J Hum Genet 51:559-566

Kokubo Y, Tomoike H, Tanaka C, Banno M, Okuda T, Inamoto N, Kamide K, Kawano Y, Miyata T (2006) Association of sixty-one non-synonymous polymorphisms in forty-one hypertension candidate genes with blood pressure variation and hypertension. Hypertens Res 29:611-619

Koo BK, Cho YM, Park BL, Cheong HS, Shin HD, Jang HC, Kim SY, Lee HK, Park KS (2007) Polymorphisms of KCNJ11 (Kir6.2 gene) are associated with Type 2 diabetes and hypertension in the Korean population. Diabet Med 24:178-186

Koster JC, Permutt MA, Nichols CG (2005) Diabetes and insulin secretion: the ATP-sensitive $\mathrm{K}+$ channel (K ATP) connection. Diabetes 54:3065-3072

Lillienfeld AM, Lillienfeld DE (1980) Foundations of epidemiology, 2nd edn. Oxford University Press, New York

Liu Z, Zhang YW, Feng QP, Li YF, Wu GD, Zuo J, Xiao XH, Fang FD (2006) Association analysis of 30 type 2 diabetes candidate genes in Chinese Han population. Zhongguo Yi Xue Ke Xue Yuan Xue Bao 28:124-128

Miki T, Liss B, Minami K, Shiuchi T, Saraya A, Kashima Y, Horiuchi M, Ashcroft F, Minokoshi Y, Roeper J, Seino S (2001) ATPsensitive $\mathrm{K}+$ channels in the hypothalamus are essential for the maintenance of glucose homeostasis. Nat Neurosci 4:507-512

Nielsen EM, Hansen L, Carstensen B, Echwald SM, Drivsholm T, Glumer C, Thorsteinsson B, Borch-Johnsen K, Hansen T, Pedersen O (2003) The E23K variant of Kir6.2 associates with impaired post-OGTT serum insulin response and increased risk of type 2 diabetes. Diabetes 52:573-577

Reimann F, Gribble FM (2002) Glucose-sensing in glucagon-like peptide-1-secreting cells. Diabetes 51:2757-2763

Riedel MJ, Boora P, Steckley D, de Vries G, Light PE (2003) Kir6.2 polymorphisms sensitize beta-cell ATP-sensitive potassium channels to activation by acyl CoAs: a possible cellular mechanism for increased susceptibility to type 2 diabetes? Diabetes 52:2630-2635

Riedel MJ, Steckley DC, Light PE (2005) Current status of the E23K Kir6.2 polymorphism: implications for type-2 diabetes. Hum Genet 116:133-145
Schwanstecher C, Meyer U, Schwanstecher M (2002a) K(IR)6.2 polymorphism predisposes to type 2 diabetes by inducing overactivity of pancreatic beta-cell ATP-sensitive $\mathrm{K}(+)$ channels. Diabetes 51:875-879

Schwanstecher C, Neugebauer B, Schulz M, Schwanstecher M (2002b) The common single nucleotide polymorphism E23K in K(IR)6.2 sensitizes pancreatic beta-cell ATP-sensitive potassium channels toward activation through nucleoside diphosphates. Diabetes 51(Suppl 3):S363-S367

Schwanstecher C, Schwanstecher M (2002) Nucleotide sensitivity of pancreatic ATP-sensitive potassium channels and type 2 diabetes. Diabetes 51(Suppl 3):S358-S362

Seino S, Miki T (2003) Physiological and pathophysiological roles of ATP-sensitive K+ channels. Prog Biophys Mol Biol 81:133-176

Storey JD, Tibshirani R (2003) Statistical significance for genomewide studies. Proc Natl Acad Sci USA 100:9440-9445

Tanahashi T, Osabe D, Nomura K, Shinohara S, Kato H, Ichiishi E, Nakamura N, Yoshikawa T, Takata Y, Miyamoto T, Shiota H, Keshavarz P, Yamaguchi Y, Kunika K, Moritani M, Inoue H, Itakura M (2006) Association study on chromosome 20q11.2113.13 locus and its contribution to type 2 diabetes susceptibility in Japanese. Hum Genet 120:527-542

Yamada Y, Kuroe A, Li Q, Someya Y, Kubota A, Ihara Y, Tsuura Y, Seino Y (2001) Genomic variation in pancreatic ion channel genes in Japanese type 2 diabetic patients. Diabetes Metab Res Rev 17:213-216

Yokoi N, Kanamori M, Horikawa Y, Takeda J, Sanke T, Furuta H, Nanjo K, Mori H, Kasuga M, Hara K, Kadowaki T, Tanizawa Y, Oka Y, Iwami Y, Ohgawara H, Yamada Y, Seino Y, Yano H, Cox NJ, Seino S (2006) Association studies of variants in the genes involved in pancreatic beta-cell function in type 2 diabetes in Japanese subjects. Diabetes 55:2379-2386

Zingman LV, Hodgson DM, Bast PH, Kane GC, Perez-Terzic C, Gumina RJ, Pucar D, Bienengraeber M, Dzeja PP, Miki T, Seino S, Alekseev AE, Terzic A (2002) Kir6.2 is required for adaptation to stress. Proc Natl Acad Sci USA 99:13278-13283 